Proceedings of SALT 28: 809-829, 2018

\title{
Do modals take propositions or sets of propositions? Evidence from Japanese darou*
}

\author{
Wataru Uegaki \\ LUCL, Leiden University
}

\author{
Floris Roelofsen \\ ILLC, University of Amsterdam
}

\begin{abstract}
The Japanese modal particle darou can take either a declarative or an interrogative prejacent (Hara 2006; Hara \& Davis 2013). We point out, however, that its interrogative-embedding use cannot be reduced to its declarative-embedding use. This is problematic under the standard assumption that modal operators always apply to propositions, but not under more recent proposals which take modal operators to apply to sets of propositions. We develop a detailed account of darou, capturing its non-reductive nature as well as its puzzling interaction with intonation (Hara 2015).
\end{abstract}

Keywords: modality; responsive predicates; clause embedding; inquisitive semantics.

\section{Introduction}

Modal operators are traditionally taken to apply to propositions. For instance, in (1) the modal predicate know is taken to apply to the proposition expressed by its declarative complement that Amy left.

(1) John knows that Amy left.

But what if know is combined with an interrogative complement, as in (2)?

(2) John knows who left.

Clause-embedding predicates that take both declarative and interrogative complements are called responsive predicates. Under the traditional view, the interrogativeembedding use of responsive predicates is reduced to their declarative-embedding use (e.g., Karttunen 1977; Heim 1994; Lahiri 2000; Spector \& Egré 2015). For instance, (2) may be analyzed as in (3):

* We thank Yurie Hara, Satoshi Tomioka, Malte Zimmermann, and audiences at the Selection Fest 2017 and SALT 28 for helpful comments and discussion. We also gratefully acknowledge financial support from the European Research Council (ERC, grant agreement number 680220) and from the Netherlands Organisation for Scientific Research (NWO, grant number IG.18.023). 
(3) For some proposition $p$ that is an answer to the question expressed by the complement who left, John knows $p$.

Thus, whether know syntactically combines with a declarative or an interrogative complement, semantically it is always taken to apply to a proposition.

Another, more recent view is that modals always apply to sets of propositions (Ciardelli \& Roelofsen 2015, 2018; Uegaki 2015; Theiler, Roelofsen \& Aloni 2018). Under this view, both declarative and interrogative complements denote sets of propositions. In the case of declarative complements this set only has one element (or, in inquisitive semantics, only one maximal element). On the other hand, in the case of interrogative complements it contains multiple propositions. For instance, in (1) know may be taken to apply to the singleton set $\{\lambda w$.Amy left in $w\}$, and in (2) it may be taken to apply to the set of propositions $\{\lambda w . d$ left in $w \mid d \in D\}$. Thus, the interrogative-embedding use of know does not need to be reduced to its declarative-embedding use; rather, it is possible to define an entry for know which applies uniformly to both types of complement.

George $(2011,2013)$ develops an argument against the reductive approach based on the observation that the truth of an interrogative knowledge ascription cannot always be characterized purely in terms of true propositional knowledge-false beliefs are sometimes also relevant. For instance, for (4) to be true it is not only required that John knows a proposition of the form 'One can get an Italian newspaper at store $x$ ' but also that he does not believe any false propositions of this form.

John knows where one can get an Italian newspaper.

Elliott, Klinedinst, Sudo \& Uegaki (2017) develop another argument against the reductive approach, involving so-called predicates of relevance like care and matter. For instance, they note that (5a) cannot be analyzed reductively as in (5b).

a. It matters to John who left.

b. 'For some answer $p$ to who left, it matters to John that $p$ '

On the other hand, George (2011) and Spector \& Egré (2015) raise a concern for the uniform, non-reductive approach, which is that it does not predict any constraints on the range of possible responsive predicate meanings. To illustrate this point, Spector \& Egré consider the fictitious verb shknow, which is equivalent to know when taking a declarative complement and equivalent to wonder when taking an interrogative complement. Under the reductive approach, such predicates are predicted not to exist in any language. On the uniform, non-reductive approach, such constraints are not directly predicted (though see Theiler et al. 2018; Uegaki to appear; Steinert-Threlkeld to appear for discussion of how certain constraints on the range of possible responsive predicates may be captured under this view). 
Do modals take propositions or sets of propositions? Evidence from Japanese darou

The present paper offers new evidence for the uniform approach, coming from the Japanese modal particle darou, which behaves roughly like Spector \& Egré's fictitious shknow. That is, when combined with a declarative clause, as in (6), darou translates as 'I believe', while in the presence of the question particle $\mathrm{ka}$, as in (7), it translates as 'I wonder' (Hara 2006; Hara \& Davis 2013; Hara 2015). ${ }^{1}$

Taro-wa utau-darou.

Taro-TOP sing-DAROU

'I believe that Taro will sing.'

\section{Dare-ga utau-darou-ka.}

who-NOM sing-DAROU-Q

'I wonder who will sing.'

Crucially, the use of darou in (7) cannot be analyzed reductively: to wonder about a question is something quite different from believing one of the answers to that question.

We will develop an account of darou which captures its semantic contribution when combined with declarative and interrogative clauses, as well as its puzzling interaction with intonation (which will be discussed in some detail below). The account crucially relies on the assumption that declarative and interrogative clauses both denote sets of propositions, and that modal operators apply uniformly to both types of complement, taking sets of propositions as their input. ${ }^{2}$

The structure of the paper is as follows. The empirical properties of darou are discussed in some detail in Section 2. The theoretical background for our proposal is given in Section 3, and the proposal itself is spelled out in Section 4. Finally, Section 5 concludes.

\section{Basic data and challenges}

In this section, we present the basic data concerning the interpretation of darou, and discuss the challenges they present.

1 Roberts (2018) discusses an attitude predicate in Estonian, mõtlema, whose semantics is similar to Japanese darou. He similarly reaches the conclusion that modal operators generally apply to sets of propositions rather than to single propositions.

2 Shortly before finalising the current paper, we became aware of Hara 2017, 2018, which lays out an account of darou which is in some ways very similar to ours, yet also differs from it in several respects. A detailed comparison between the two accounts is left for future work, but we will highlight some salient differences in footnote 8. 
Uegaki and Roelofsen

\subsection{Darou is a responsive modal particle}

Our starting point is the observation that darou can take either a declarative or an interrogative clause as its prejacent. This is shown in the following examples, where (8) involves a declarative clause and (9) involves interrogative clauses (a polar question for (9a) and a wh-question for (9b)):

$$
\text { Taro-ga utau-darou } \downarrow \text {. }
$$

Taro-NOM sing-DAROU

'I believe Taro will sing.'
a. Taro-ga utau-darou-ka $\downarrow$. Taro-NOM sing-DAROU-Q
'I wonder if Taro will sing.'
b. Dare-ga utau-darou-ka $\downarrow$. who-NOM sing-DAROU-Q
'I wonder who will sing.'

This means that darou is a responsive modal particle, i.e., a particle that can take either a declarative or an interrogative prejacent. Roughly, darou contributes a meaning similar to 'I believe' when it embeds a declarative prejacent, as in (8), and a meaning similar to 'I wonder' when it embeds an interrogative prejacent, as in (9). We will refine the description of these interpretations as we discuss more data below. ${ }^{3}$

Regarding the darou-ka sentences in (9), following Hara (2006, 2015), we treat the interrogative clause headed by the question particle $k a$ as scoping below darou, despite the surface order in which $k a$ follows darou. That is, we take the sentences in (9) to have the following kind of LF structures:
a. [[Taro sing]-KA]-DAROU
b. [[who sing]-KA]-DAROU

There are several arguments supporting this semantic treatment of darou-ka sentences. The first argument comes from intonation. The sentences require a sentencefinal falling intonation, as indicated in (9), and are incompatible with a sentence-

3 Another important aspect of the interpretation of darou is that it is an evidential particle, whose use is constrained by the nature of the 'evidence' the speaker has for the relevant belief. Based on observations in the earlier literature (Morimoto 1994; Takubo 2001; Sugimura 2004), Hara (2006) argues that $p$-darou is felicitous only if the speaker 'has an epistemic bias for $p$ derived from reasoning and not from observable (direct or indirect) evidence' (Hara 2006: 133-4). We will largely set aside the evidential aspect of darou in this paper, focusing on the compositional semantics of darou with different clause types. In footnote 5, we suggest a way to capture the evidential meaning of darou by fixing the 'modal base' appropriately, following Hara (2006). 
Do modals take propositions or sets of propositions? Evidence from Japanese darou

final rise. This would be puzzling if $k a$ were to scope over the entire sentences, inducing a matrix question interpretation. This is so because a matrix question ending with $k a$ requires rising intonation, as exemplified below:

$$
\begin{aligned}
& \text { Taro-wa utai-masu ka } \uparrow \\
& \text { Taro-TOP sing-POLITE KA } \\
& \text { 'Will Taro sing?' }
\end{aligned}
$$

(polar question)

Thus, the intonation of the darou- $k a$ sentences in (9) is unexpected if $k a$ scopes over the entire sentence to form a matrix question.

In addition to the intonational evidence, there is also semantic evidence suggesting that the darou-ka sentences in (9) are not matrix questions. In (12) below, it is shown that one cannot respond to the darou-ka sentences with 'Why are you asking me that question?':
A: $\quad\{$ Taro-wa / dare-ga $\}$ utau-darou-ka $\downarrow$.
B: \#Nande watashi-ni sonna koto kiku-no?
$(=(9 a) /(9 b))$ why I-DAT such thing ask-Q
'Why do you ask me such a thing?'

Furthermore, in (13) below, it is shown that a darou-ka clause cannot be used as a response to 'What do you want to know?' while a corresponding clause without darou can be used for this purpose:
A: Nani-o siri-tagat-teiru-no what-ACC know-want-ASP-Q
'What do you want to know?'

B: Watashi-ga shiri-tai-no-wa Taro/dare-ga

I-NOM know-want-GEN-TOP Taro/who-NOM

utau-(\#darou-)ka-desu. sing-DAROU-KA-COP

'What I want to know is \{ whether (I believe) Taro will sing / who will sing \}.' (intended)

These observations point to the conclusion that the darou-ka sentences do not have question interpretations that are expected if $k a$ scoped over darou.

At this point, we should acknowledge the fact that the question interpretation is not the only interpretation available for a sentence with $k a$ with matrix scope. A $k a$-ending sentence can have an exclamative interpretation with a sentence-final fall, as shown below:

$$
\text { Taro-wa utai-masu ka } \downarrow
$$


However, it is evident that the sentences in (9) do not have exclamative interpretations along the same lines as (14). For example, (9a) does not have the interpretation 'It is surprising that I believe that Taro will sing!'. In particular, such an exclamative interpretation would entail the speaker's belief that Taro will sing, contrary to the fact that (9a) conveys the speaker's ignorance about whether Taro will sing. Thus, the interpretations of darou-ka sentences cannot be analyzed as a result of the matrix scope of $k a$, whether $k a$ contributes a question interpretation or an exclamative interpretation. In our analysis presented below, we will argue that the empirically correct interpretation of darou-ka sentences can be derived by assuming that the question particle $k a$ scopes below darou.

All in all, the above observations suggest that darou is a responsive modal particle, i.e., it can take either a declarative or an interrogative prejacent.

\subsection{Darou is non-reducible}

Given that darou is a responsive modal particle, a question arises as to whether we can adopt the reductive view in analyzing its meaning. That is, whether the semantic contribution of darou with interrogative prejacents can be reduced to that with declarative prejacents.

The interpretations observed in (8-9) already suggest that darou is not amenable to a reductive treatment. To see this, let us consider a reductive account of darou in terms of a 'mention-some' reading, i.e., existential quantification over possible answers to the question:

\section{A reductive treatment of darou (mention-some) ' $Q$-darou' iff for some answer $p$ to $Q$, ' $p$-darou'}

This analysis is clearly incorrect because the interpretation of $Q$-darou, i.e., 'I wonder Q', does not mean that for some answer $p$ to $Q, p$-darou, i.e., 'I believe $p$ '. In fact, (9) conveys the speaker's ignorance about the answer to the question.

Now, existing reductive accounts of responsive predicates are more sophisticated than the simple existential semantics sketched in (15). In particular, Karttunen 1977 and much subsequent work employ (some version of) mention-all answers, treating $Q$-darou as having a stronger interpretation than (15), as follows:

\section{A reductive treatment of darou (mention-all)} ' $Q$-darou' iff for all true answers $p$ to $Q$, ' $p$-darou'

This too is clearly incorrect, again, as $Q$-darou does not entail that the speaker believes any specific answer to the question. In fact, all existing reductive accounts 
Do modals take propositions or sets of propositions? Evidence from Japanese darou

employ a notion of answerhood that is at least as strong as the mention-some answer. Thus, these accounts suffer from the same problem as the incorrect prediction of the mention-some account in (15).

A more general argument for the non-reductive nature of darou, which does not only target all existing reductive approaches, but really any conceivable such account, goes as follows. Suppose Hanako and Mari are going to a wedding. They both know that some of the wedding guests will sing for the bride and groome. More specifically, they both believe that Taro will sing. Of all the other guests they don't have any expectations as to whether they will sing or not. Hanako would like to know who exactly will sing. Mari on the other hand is not really interested. In this scenario, both Hanako and Mari can truthfully utter (8), since they both expect that Taro will sing. Moreover, neither Hanako nor Mari can truthfully utter sentences which are obtained from (8) by replacing Taro by any other wedding guest. So, when it comes to darou sentences with declarative prejacents about who will sing at the wedding, exactly the same sentences can be truthfully uttered by Hanako and Mari. And yet, only Hanako can truthfully utter (9b) in this scenario, because only she is interested in who will sing. This makes a reductive account of darou sentences with interrogative prejacents impossible, no matter how this reduction is defined exactly.

Hence, darou is a non-reductive modal particle, which puts in the same class as other modal operators that have been shown to require a non-reductive treatment, i.e., 'false-answer-sensitive' predicates such as know and forget (George 2011), predicates of relevance such as care and matter (Elliott et al. 2017), and the Estonian epistemic-inquisitive attitude predicate mõtlema (Roberts 2018). In §4, we will propose a non-reductive analysis of darou.

\subsection{Effects of intonation}

What makes darou even more interesting is its interaction with intonation (Hara 2015). As seen in (17), with final rising intonation, darou with a declarative prejacent expresses a biased question, similar to English tag-questions. On the other hand, as briefly mentioned above, rising intonation is incompatible with darou-ka, as in (18).

Taro-wa utau-darou $\uparrow$.

'Taro will sing, won't he?'

a. *Taro-wa utau-darou-ka $\uparrow$.

b. *Dare-ga utau-darou-ka $\uparrow$.

Thus, rising intonation is compatible with darou when it embeds a declarative pre- 
jacent but not when it embeds an interrogative prejacent. A semantic analysis of darou has to account for this fact, in addition to its non-reductive nature. In the analysis to be presented in $\S 4$, we will propose a concrete analysis of how intonation interacts compositionally with darou and $\mathrm{ka}$, and why certain combinations turn out to be unacceptable given pragmatic considerations.

\section{Theoretical background}

Our analysis of darou will be couched in inquisitive epistemic logic (IEL; Ciardelli \& Roelofsen 2015). In IEL, declarative and interrogative clauses are taken to have the same kind of semantic value, i.e., sets of propositions. Modal operators are assumed to take such sets of propositions as their input. This makes it possible to account for non-reducible responsive modal particles like darou. In this section, we briefly specify the formal models assumed in IEL and the semantics of its logical operators. This will form the basis for our analysis of darou, $k a$ and the relevant intonation patterns to be presented in $\$ 4$.

Hara $(2017,2018)$ has independently developed a comparable IEL analysis of darou, making use of the fact that modals in IEL take sets of propositions to capture the behavior of darou as a responsive modal particle (see also footnotes 2 and 8).

\subsection{Models}

An IEL model for a set $\mathscr{P}$ of atomic sentences and a set $\mathscr{A}$ of agents is a triple $M=\left\langle\mathscr{W}, V, \mathrm{INQ}_{\mathscr{A}}\right\rangle$, where:

a. $\quad \mathscr{W}$ is a set of POSSIBLE WORLDS,

b. $\quad V: \mathscr{W} \mapsto \wp(\mathscr{P})$ is a VALUATION MAP, and

c. $\quad \mathrm{INQ}_{\mathscr{A}}=\left\{\mathrm{INQ}_{a} \mid a \in \mathscr{A}\right\}$ is a set of INQUISITIVE STATE MAPS, one for each agent $a \in \mathscr{A}$, mapping every world $w \in \mathscr{W}$ to a downward-closed set of information states $\mathrm{INQ}_{a}(w)$, namely those information states that settle the issues that $a$ entertains in $w$.

Here, $\operatorname{INQ}_{a}(w)$ is called the INQUISITIVE STATE of $a$ in $w$, and its union, $\bigcup \operatorname{INQ}_{a}(w)$, represents the current information state of $a$ in $w$, i.e., $\operatorname{DOX}_{a}(w)$ in classical Hintikkan epistemic logic. That is, we have that $\bigcup \operatorname{INQ}_{a}(w)=\operatorname{DOX}_{a}(w)$. For further discussion of IEL models we refer to Ciardelli \& Roelofsen 2015; Ciardelli 2016.

\subsection{Semantics}

The semantic value of a sentence $\varphi$ in IEL, $\llbracket \varphi \rrbracket$, is a downward-closed set of propositions, namely those propositions that support the information conveyed by $\varphi$ (if 
Do modals take propositions or sets of propositions? Evidence from Japanese darou

any) and resolve the issue expressed by $\varphi$ (if any). The truth-conditions of $\varphi$ are also derivable from $\llbracket \varphi \rrbracket$ as follows:

(19) For any sentence $\varphi$ and world $w, \varphi$ is true in $w$ iff $\{w\} \in \llbracket \varphi \rrbracket$.

The set of all worlds where $\varphi$ is true is referred to as the INFORMATIVE CONTENT of $\varphi$, written as $\operatorname{info}(\varphi)$. Also, the maximal elements of $\llbracket \varphi \rrbracket$ are referred to as the ALTERNATIVES in $\llbracket \varphi \rrbracket$, written as $\operatorname{alt}(\varphi)$.

For any sentence $\varphi$,

a. $\operatorname{info}(\varphi):=\bigcup \llbracket \varphi \rrbracket$

b. $\quad \operatorname{alt}(\varphi):=\{p \in \llbracket \varphi \rrbracket \mid$ there is no $q \in \llbracket \varphi \rrbracket$ such that $p \subset q\}$

We say that $\varphi$ is INQUISITIVE if $|\operatorname{alt}(\varphi)| \geq 2$, and NON-INQUISITIVE if $\mid$ alt $(\varphi) \mid=1$.

Given this setup, the semantics of the basic connectives and operators in IEL can be defined as follows:

(21) For any atomic sentence $\alpha$, any atomic or non-atomic sentences $\varphi, \psi$, and any agent $a$,
a. $\llbracket \alpha \rrbracket:=\{p \mid \forall w \in p: \alpha \in V(w)\}$
b. $\quad \llbracket \neg \varphi \rrbracket:=\left\{p \mid \forall p^{\prime} \in \llbracket \varphi \rrbracket: p \cap p^{\prime}=\varnothing\right\}$
c. $\llbracket \varphi \wedge \psi \rrbracket:=\llbracket \varphi \rrbracket \cap \llbracket \psi \rrbracket$
d. $\quad \llbracket B_{a} \varphi \rrbracket:=\left\{p \mid \forall w \in p: \operatorname{DOX}_{a}(w) \in \llbracket \varphi \rrbracket\right\}$
e. $\left.\quad \llbracket E_{a} \varphi \rrbracket:=\left\{p \mid \forall w \in p: \operatorname{INQ}_{a}(w) \subseteq \llbracket \varphi \rrbracket\right)\right\}$

Here, $B$ and $E$ are modal operators referred to as the 'BELIEVE'-MODALITY and the 'ENTERTAIN'-MODALITY, respectively. $B_{a} \varphi$ is similar to the epistemic modality in classical epistemic logic in that it requires $a$ 's current information state to support the information conveyed by $\varphi$. On top of this, however, it also requires $a$ 's current information state to resolve the issue expressed by $\varphi$ (if any). On the other hand, $E_{a} \varphi$ states that if the issues that $a$ entertains were to be settled, the issue expressed by $\varphi$ would also be settled. What is important for our purposes is that the semantics of the 'entertain'-modality is non-reductive, i.e., its meaning cannot be expressed in terms of a relationship between an agent and a single proposition. We will make crucial use of the 'entertain'-modality to analyze the non-reductive meaning of darou.

As an illustration, it is instructive to mention the semantics for the English predicate wonder in IEL suggested by Ciardelli \& Roelofsen (2015), which is as follows:

$$
\llbracket a \text { wonders } \varphi \rrbracket=\neg B_{a} \varphi \wedge E_{a} \varphi
$$

That is, $a$ wonders $\varphi$ iff $a$ 's current information state does not resolve $\varphi$ but $a$ does 
entertain the issue expressed by $\varphi$.

Another important fact about the $B$-modality and the $E$-modality we will utilize is that they are equivalent if their prejacent is non-inquisitive. That is:

$$
\text { For any sentence } \varphi \text { and agent } a \text {, if }|\operatorname{alt}(\varphi)|=1 \text {, then } \llbracket B_{a} \varphi \rrbracket=\llbracket E_{a} \varphi \rrbracket \text {. }
$$

Here is why. If $|\operatorname{alt}(\varphi)|=1$, then $\llbracket \varphi \rrbracket=\{p \mid p \subseteq \operatorname{info}(\varphi)\}$. This means that:

$$
\begin{aligned}
& \llbracket B_{a} \varphi \rrbracket=\left\{p \mid \forall w \in p: \operatorname{DOX}_{a}(w) \subseteq \operatorname{info}(\varphi)\right\}
\end{aligned}
$$

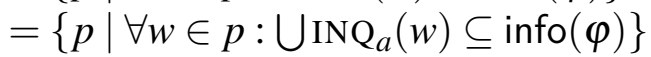

$$
\begin{aligned}
& =\left\{p \mid \forall w \in p: \operatorname{INQ}_{a}(w) \subseteq\{p \mid p \subseteq \operatorname{info}(\varphi)\}\right\} \\
& =\llbracket E_{a} \varphi \rrbracket
\end{aligned}
$$

A corollary of this is that (22) ends up being a contradiction when $\varphi$ is noninquisitive, explaining the fact that wonder is incompatible with declarative complements (Ciardelli \& Roelofsen 2015; cf. Uegaki 2015).

Additionally, we will make use of two operators, ! and $\langle ?\rangle$, defined as follows:

$$
\begin{array}{ll}
\text { a. } & \llbracket ! \varphi \rrbracket:=\{p \mid p \subseteq \operatorname{info}(\varphi)\} \\
\text { b. } & \llbracket\langle ?\rangle \varphi \rrbracket:=\left\{\begin{array}{ll}
\llbracket \varphi \rrbracket \cup \llbracket \neg \varphi \rrbracket & \text { if }|\operatorname{alt}(\varphi)|=1 \\
\llbracket \varphi \rrbracket & \text { if }|\operatorname{alt}(\varphi)| \geq 2
\end{array}\right\}
\end{array}
$$

These operators have the role of ensuring non-inquisitiveness and inquisitiveness, respectively. That is, for any $\varphi, ! \varphi$ is non-inquisitive and $\langle ?\rangle \varphi$ is inquisitive. ${ }^{4}$

\section{Proposal}

We are now in a position to spell out our semantic analysis of darou and illustrate how the analysis captures the facts presented in $\S 2$.

\subsection{Formal treatment of darou, $k a$ and sentence-final fall/rise}

Following Hara (2015), our analysis employs a two-dimensional semantics with atissue semantic values $\llbracket \cdot \rrbracket$ and non-at-issue semantic values $\llbracket \cdot \rrbracket^{\bullet}$. In this subsection, we will present the lexical entries for the crucial vocabularies to capture our data, i.e., darou, $k a$ and the sentence-final rising and falling pitch contours (i.e., $\uparrow$ and $\downarrow$ ). An analysis of concrete examples using these lexical entries will be given in the next subsection.

4 We employ the conditional definition of the operator $\langle ?\rangle$ from Roelofsen \& Farkas 2015 instead of the more basic operator ? from Ciardelli \& Roelofsen 2015, defined as $\llbracket \varphi \rrbracket:=\llbracket \varphi \rrbracket \cup \llbracket \neg \varphi \rrbracket$. This is to ensure that the operator makes an appropriate semantic contribution when taken as the question operator in the case of $w h$-questions, as will be detailed in $\S 4$. 
Do modals take propositions or sets of propositions? Evidence from Japanese darou

First, we analyze darou as follows:

$$
\begin{array}{ll}
\text { a. } & \llbracket \varphi \text { darou } \rrbracket=\llbracket\langle ?\rangle ! \varphi \rrbracket \\
\text { b. } & \llbracket \varphi \text { darou } \rrbracket^{\bullet}=\llbracket E_{\odot} \varphi \rrbracket \cap \llbracket \varphi \rrbracket
\end{array}
$$

(where $\odot$ is the contextually determined deictic center; Hara 2006)

According to this semantics, $\varphi$-darou has the question 'whether-! $\varphi$ ' as its at-issue meaning, and conveys that 'the deictic center entertains $\varphi$ ' as its non-at-issue meaning. As we will see in the next section, the 'entertain' modality allows us to properly capture the non-reductive meaning of darou. 5

The deictic center $\odot$ is normally the speaker in matrix sentences. However, the following example by Hara (2006: 128-9) shows that the center can be shifted to the attitude holder in a reported attitude:

$$
\begin{aligned}
& \text { Hanako-wa [ Taro-ga kuru darou to ] omot-teiru. Boku-wa } \\
& \text { Hanako-TOP [ Taro-NOM come DAROU COMP ] think-ASP. I-TOP } \\
& \text { sou-wa omow-anai-kedo. } \\
& \text { so-TOP think-NEG-though. } \\
& \text { 'Hanako thinks that Taro will come-DAROU, but I don't think so' }
\end{aligned}
$$

In this paper, we will be silent about the exact mechanism determining the deictic center in a given context, as the issue is independent from our primary purpose, i.e., to give a uniform semantic treatment for the interpretation of darou with declarative and interrogative prejacents.

The lexical entries for the sentence-final pitch contours and $k a$ are as follows:
a. $\llbracket \varphi \downarrow \rrbracket=\llbracket ! \varphi \rrbracket$
b. $\quad \llbracket \varphi \downarrow \rrbracket^{\bullet}=\llbracket \varphi \rrbracket^{\bullet}$
a. $\llbracket \varphi \uparrow \rrbracket=\llbracket\langle ?\rangle \varphi \rrbracket$
b. $\llbracket \varphi \uparrow \rrbracket^{\bullet}=\llbracket \varphi \rrbracket^{\bullet}$
a. $\quad \llbracket \varphi \mathrm{ka} \rrbracket=\llbracket\langle ?\rangle \varphi \rrbracket$
b. $\quad \llbracket \varphi \mathrm{ka} \rrbracket^{\bullet}=\llbracket \varphi \rrbracket^{\bullet}$

The non-at-issue semantic contribution of $k a$ and the pitch contours is trivial. In the at-issue dimension, the fall $\downarrow$ contributes a ! operator while both the rise $\uparrow$ and $k a$ contribute $\langle ?\rangle$.

Independently of the analysis of darou, the entries in (28-30) make correct predictions about the interpretation of $k a$ and sentence-final intonation contours with a non-inquisitive prejacent, such as the following:

5 Hara (2006) analyzes the evidential nature of darou (see footnote 3) by fixing the modal base appropriately in the Kratzerian analysis of modality (Kratzer 1981). Following this, we can assume that the lexical semantics of darou involves slight variants of $\operatorname{DOX}_{a}(w)$ and $\operatorname{INQ}_{a}(w)$ : $\operatorname{DOX}_{a}^{\prime}(w)$, the set of worlds compatible with $a$ 's reasoning in $w$ not based on observable evidence, and $\mathrm{INQ}_{a}^{\prime}(w)$, the downward-closed set of information states that settle the issues within $\operatorname{DOX}_{a}^{\prime}(w)$ that $a$ entertains in $w$. The structure of the analysis presented below will be the same under such an analysis. 

a. Taro-wa utai-masu $\downarrow$
Taro-TOP sing-POL
'Taro will sing.'
b. Taro-wa utai-masu $\uparrow$
'Will Taro sing?'
c. Taro-wa utai-masu-ka $\uparrow$
'Will Taro sing?'

In (31), we see that a non-inquisitive prejacent with a fall expresses a plain declarative statement. On the other hand, a non-inquisitive prejacent with either just a rise or with $k a$ and a rise expresses a polar question. This is correctly captured by the entries in (28-30), as shown below, where $\llbracket \alpha \rrbracket=\llbracket$ Taro will sing

$$
\begin{array}{ll}
\text { a. } & \llbracket(31 \mathrm{a}) \rrbracket=\llbracket ! \alpha \rrbracket=\llbracket \alpha \rrbracket \\
\text { b. } & \llbracket(31 \mathrm{~b}) \rrbracket=\llbracket\langle ?\rangle \alpha \rrbracket=\llbracket \alpha \rrbracket \cup \llbracket \neg \alpha \rrbracket \\
\text { c. } & \llbracket(31 \mathrm{c}) \rrbracket=\llbracket\langle ?\rangle\langle ?\rangle \alpha \rrbracket=\llbracket\langle ?\rangle \alpha \rrbracket=\llbracket \alpha \rrbracket \cup \llbracket \neg \alpha \rrbracket
\end{array}
$$

The analysis also makes correct predictions for inquisitive prejacents that involve a $w h$-phrase, but we will defer the illustration of this to $\$ 4.2 .2$, where we discuss the compositional semantics of $w h$-clauses in some detail.

We should mention at this point that the exclamative use of $k a$ briefly discussed in $\S 2$, as exemplified below, is not captured in the current analysis.

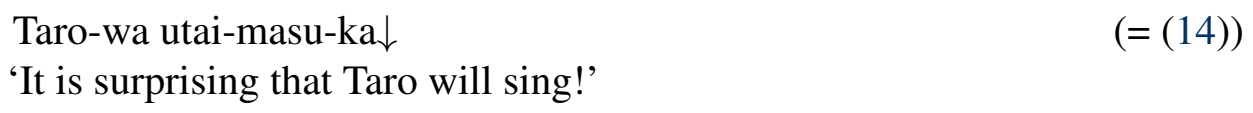$$
(=(14))
$$

Simply applying the entries in (28-30) to (33) would predict that (33) has a tautologous at-issue meaning, $\llbracket !\langle ?\rangle \alpha \rrbracket$. On the other hand, the non-at-issue meaning would be equivalent to the at-issue meaning of the prejacent, i.e., $\llbracket \alpha \rrbracket$. It is left as an open issue whether the exclamative interpretation can be derived from such a pair of semantic values as a result of pragmatic reasoning, or whether the phenomenon forces us to posit a distinct lexical entry for $k a$ or $\downarrow$.

\subsection{Analysis of concrete examples}

Given the lexical entries for darou, $k a$ and the sentence-final pitch contours, we can account for the interpretations of the basic examples given in $\S 2$. For expository purposes, we will start the discussion with cases in which the prejacent of darou is a declarative clause or a polar interrogative clause (\$4.2.1). The analysis will be expanded in $\$ 4.2 .2$ to deal with cases involving $w h$-prejacents. 
Do modals take propositions or sets of propositions? Evidence from Japanese darou

\subsubsection{Non-wh cases}

In the following, we let $\alpha$ be a clause like Taro-wa utau 'Taro will sing' which does not involve a $w h$-phrase or other alternative-inducing items. Also, in the following, we write $\llbracket \alpha \rrbracket$ for $\llbracket ! \alpha \rrbracket$ because $\llbracket ! \alpha \rrbracket=\llbracket \alpha \rrbracket$ given the non-inquisitiveness of $\llbracket \alpha \rrbracket$.

Non-wh-clause + darou $\downarrow \quad$ As discussed in $\S 2$, when darou embeds a declarative clause and is accompanied by a fall, it contributes a meaning close to 'I believe'. This is shown in the following example repeated from (8):

$$
\begin{aligned}
& \text { Taro-wa utau-darou } \downarrow \text {. } \\
& \text { Taro-TOP sing-DAROU } \\
& \text { 'I believe Taro will sing.' }
\end{aligned}
$$

The current analysis predicts that (34) has the following pair of semantic values:

$$
\begin{array}{ll}
\text { a. } & \llbracket \alpha \text { darou } \downarrow \rrbracket=\llbracket !\langle ?\rangle \alpha \rrbracket \quad \text { (tautologous) } \\
\text { b. } \quad \llbracket \alpha \text { darou } \downarrow \rrbracket^{\bullet}=\llbracket E_{\odot} \alpha \rrbracket=\llbracket B_{\odot} \alpha \rrbracket
\end{array}
$$

The at-issue meaning in (35a) is tautologous because $\llbracket !\langle ?\rangle \alpha \rrbracket=\wp(\bigcup(\llbracket \alpha \rrbracket \cup \llbracket \neg \alpha \rrbracket))$. The $E$-modality in the non-at-issue meaning in (35b) boils down to the $B$-modality because the prejacent is non-inquisitive. Altogether, (34) is predicted to convey the speaker's belief that Taro will sing as its non-at-issue meaning.

This aligns with Hara's $(2006,2015)$ claim that the speaker's attitude conveyed by darou is non-at-issue. Hara (2006) supports this claim by Sugimura's (2004) observation that darou cannot be embedded under a sentential negation. This is exemplified in the following:

$$
\begin{aligned}
& \text { ??Taro-wa utau-darou (toiu wake-de-wa) nai } \\
& \text { Taro-TOP sing-DAROU COMP NOMINALIZER-COP-TOP NEG } \\
& \text { 'It is not the case that I believe Taro will sing.' (intended) }
\end{aligned}
$$

The unacceptability of (36) is expected in the current analysis. Under the analysis, the non-at-issue content of the darou-clause projects out of the negation, and the negation only scopes over the tautologous at-issue content. As a result, (36) would have the same pair of semantic values as the sentence without the negation in (34). We argue that (36) would thus be blocked by (34) under semantic equivalence.

Further evidence for the non-at-issueness of the attitude meaning of darou comes from the (in)felicity of the following kind of discourse:

$$
\begin{aligned}
& \text { A: Kimi-wa boku-to doko-ga iken-ga chigau-no? } \\
& \text { you-TOP I-with where-NOM opinion-NOM different-Q } \\
& \text { 'Where do you disagree with me?' }
\end{aligned}
$$




\section{B: \#Taro-wa utau-darou. \\ 'I believe Taro will sing.' (intended) \\ B': $\checkmark$ boku-wa Taro-ga utau-to omou. \\ I-TOP Taro-NOM sing-COMP think. \\ 'I believe Taro will sing'}

The infelicity of the response in B in (37) shows that a darou-sentence cannot be used to resolve a question about the content of the speaker's belief. This contrasts with the felicitous response in B', which involves an explicit belief report. This phenomenon is expected under the analysis where the attitude meaning of darou is a non-at-issue content.

Non-wh-clause $+\boldsymbol{d a r o u} \uparrow \quad$ Next, let us consider the case where darou embeds a declarative prejacent with a rise. As in the following example repeated from (17), such a sentence conveys a meaning similar to an English tag-question:

Taro-wa utau-darou $\uparrow$.

'Taro will sing, won't he.'

The current analysis derives the following semantic values:

$$
\begin{array}{ll}
\text { a. } & \llbracket \alpha \text { darou } \uparrow \rrbracket=\llbracket\langle ?\rangle\langle ?\rangle \alpha \rrbracket=\llbracket\langle ?\rangle \alpha \rrbracket \\
\text { b. } \quad \llbracket \alpha \text { darou } \uparrow \rrbracket & =\llbracket E_{\odot} \alpha \rrbracket=\llbracket B_{\odot} \alpha \rrbracket
\end{array}
$$

In the at-issue dimension, the sentence expresses a polar question whether Taro will sing, while in the non-at-issue dimension, it conveys the belief that Taro will sing. We claim that the at-issue meaning captures the main polar-question interpretation of (38) while the belief in the non-at-issue meaning captures the bias toward the proposition expressed by the prejacent.

Non-wh-clause + darou- $k \boldsymbol{k} \downarrow \quad$ Turning now to darou-ka sentences, recall from $\S 2$ that darou in these sentences contributes the meaning of 'wonder', as shown in the following example, repeated from (9a).

$$
\begin{aligned}
& \text { Taro-wa utau-darou-ka } \downarrow \text {. } \\
& \text { Taro-TOP sing-DAROU-Q } \\
& \text { 'I wonder if Taro will sing.' }
\end{aligned}
$$

The current analysis derives the following semantic values for this type of example. Here, as argued in $\S 2, k a$ crucially scopes below darou.
a. $\llbracket \alpha$ darou-ka $\downarrow \rrbracket=\llbracket \underset{\downarrow}{!}\langle ?\rangle !\langle ? \underbrace{\langle l}_{\text {darou }}\langle ?\rangle \alpha \rrbracket \quad$ (tautologous)
b. $\quad \llbracket \alpha$ darou-ka $\downarrow \rrbracket^{\bullet}=\llbracket E_{\odot}\langle ?\rangle \alpha \rrbracket$ 
Do modals take propositions or sets of propositions? Evidence from Japanese darou

Just as in the case of $\alpha$-darou $\downarrow$, the at-issue meaning is predicted to be tautologous because $\llbracket !\langle ?\rangle \alpha \rrbracket$ is tautologous (and neither ! nor $\langle ?\rangle$ can turn a tautologous meaning into a non-tautologous one). On the other hand, the non-at-issue meaning conveys that the speaker entertains the issue whether $\alpha$. Although this is semantically compatible with the speaker believing either $\alpha$ or $\neg \alpha$, it implicates that the speaker is ignorant due to the presence of stronger alternatives: $\llbracket \alpha$ darou $\downarrow \rrbracket$ and $\llbracket \neg \alpha$ darou $\downarrow \rrbracket$. $^{6}$ Taking into account this implicature, we derive a non-at-issue interpretation similar to that of 'wonder' (i.e., entertainment plus ignorance).

In parallel to the argument given above for the non-at-issueness of the belief implication of darou-sentences, we can also give empirical evidence for the non-atissueness of the 'wonder' implication of darou-ka sentences. Such evidence comes from the (in)felicity of the following discourse:
A: 'What do you want to know?'
B: \#Taro-wa utau-darou-ka $\downarrow$.
'I wonder whether Taro will sing.'
$\mathrm{B}^{\prime}$ : Watashi-ga shiri-tai-no-wa Taro-ga utau-ka-desu.
I-NOM know-want-GEN-TOP Taro-NOM sing-KA-COP
'What I want to know is whether Taro will sing.'

The infelicity of B in (42) suggests that a darou-ka sentence cannot directly address a question as to what the speaker is wondering about. This contrasts with the felicitous response in $\mathrm{B}^{\prime}$, which involves an explicit attitude report.

Non-wh-clause + darou-ka $\uparrow \quad$ Finally, as illustrated in the following example repeated from (18a), darou-ka with a rise is unacceptable:

$$
\text { *Taro-wa utau-darou-ka } \uparrow \text {. }
$$

This fact also receives a natural explanation in the current analysis, which derives the following semantic values for (43):

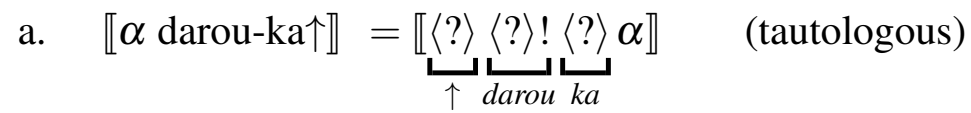

$$
\begin{aligned}
& \text { b. } \quad \llbracket \alpha \text { darou-ka } \uparrow \rrbracket^{\bullet}=\llbracket E_{\odot} ? \alpha \rrbracket
\end{aligned}
$$

Note that the at-issue and the non-at-issue semantic values in (44) are exactly the

6 Under the assumption that formal alternatives that are negated in the implicature calculation are structurally simpler than the original sentence (e.g., Katzir 2007), the analysis requires that $\alpha$-darou $\downarrow$

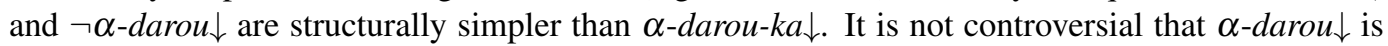
simpler than $\alpha$-darou-ka $\downarrow$. However, we do not have an independent argument to assume that $\neg \alpha$ darou $\downarrow$, or concretely, examples like Taro-wa utaw-anai-darou $\downarrow$ (Taro-TOP sing-NEG-DAROU), are simpler than $\alpha$-darou-ka $\downarrow$. This is left as an open issue. 
same as those in (41). This means that the two darou-ka sentences in (40) and (43), which only differ in their sentence-final pitch contour, are predicted to be semantically equivalent. Following e.g., Horn (1984), Levinson (2000) and Blutner (2000), we assume that this situation leads to blocking of the more marked sentence type to express the given meaning. We assume that, other things being equal, a sentence-final rise is more marked than a sentence-final fall, since in the course of pronouncing a sentence a speaker's lung pressure drops, which naturally results in a falling pitch contour (Gussenhoven 2002; Westera 2017). Given this markedness assumption, (40) blocks (43), explaining the unacceptability of the latter.

\subsubsection{Wh-cases}

We now move on to the $w h$-cases. In order to treat the semantics of $w h$-clauses, we first have to introduce presuppositions into the system. Here, we model the presupposition of a clause $\varphi$ as a proposition (a set of worlds) and denote it as $\operatorname{presup}(\varphi)$. We can then represent the interpretation of $w$ h-clauses as follows:

$$
\begin{array}{ll}
\text { a. } & \llbracket \text { dare-ga utau } \rrbracket=\{p \mid \exists d \in D: \forall w \in p: d \text { will sing in } w\} \\
\text { b. } & \llbracket \text { dare-ga utau } \rrbracket^{\bullet}=\{p \mid \exists d \in D: \forall w \in p: d \text { will sing in } w\} \\
\text { c. } & \text { presup(dare-ga utau) }=\{w \mid \exists d \in D: d \text { will sing in } w\}
\end{array}
$$

As seen in (45), both the at-issue and the non-at-issue meanings of a $w h$-clause are the downward closure of the Hamblin 1973-style denotation. The presupposition of a wh-clause, on the other hand, states that at least one of the alternatives of such a denotation is true (e.g., Karttunen \& Peters 1976; Dayal 1996).

We treat the two sentential operators ! and $\langle ?\rangle$ as presupposition 'holes' in the sense of Karttunen \& Peters (1979). That is, we have the following:

$$
\operatorname{presup}(! \varphi)=\operatorname{presup}(\langle ?\rangle \varphi)=\operatorname{presup}(\varphi)
$$

As a consequence of this, applying ! to a wh-complement leads to a tautologous content relative to its presupposition. This is so since such a sentence is non-inquisitive and is also non-informative relative to the presupposition. This is seen in the following example:

$$
\begin{aligned}
\text { a. } \quad \llbracket ![\text { dare-ga utau] }] & =\wp \bigcup\{p \mid \exists d \in D: \forall w \in p: d \text { will sing in } w\} \\
& =\wp\{w \mid \exists d \in D: d \text { will sing in } w\} \\
\text { b. } \quad & \text { presup(![dare-ga utau]) }=\{w \mid \exists d \in D: d \text { will sing in } w\}
\end{aligned}
$$

This analysis is compatible with the distribution of $k a$ and the intonation patterns in wh-questions without darou. Empirically, a wh-clause can be interpreted as an information-seeking question if it ends with a rise, with or without $k a$. This is 
Do modals take propositions or sets of propositions? Evidence from Japanese darou

expected since $\langle ?\rangle$ contributed by the rise simply passes up the inquisitive at-issue content of the $w h$-clause, and an additional $\langle ?\rangle$ contributed by $k a$ has no effect. If a wh-clause ends with a fall, it cannot be interpreted as an information-seeking question. This is expected since ! contributed by the fall would derive a tautologous at-issue content. We now move on to the analysis of wh+darou sentences.

Wh-clause + $\operatorname{darou}(-k a) \downarrow \quad$ As long as the sentence ends with a falling intonation, darou can be attached to a $w h$-clause with or without the question particle $k a$, as in the following example: ${ }^{7}$

$$
\begin{aligned}
& \text { Dare-ga utau darou(-ka) } \downarrow \\
& \text { who-NOM sing DAROU-KA } \\
& \text { 'I wonder who will sing.' }
\end{aligned}
$$

Regardless of the presence of $k a$, the same interpretation arises, i.e., the speaker wonders who will sing.

This is correctly captured in the current analysis. We predict the following semantic values for (48) without $k a$.

$$
\begin{array}{lll}
\text { a. } & \text { ddare-ga utau darou } \downarrow \rrbracket=\llbracket !\langle ?\rangle ![\text { dare-ga utau }] \rrbracket \quad \text { (tautologous) } \\
\text { b. } \quad \llbracket \text { dare-ga utau darou } \downarrow \rrbracket & =\llbracket E_{\odot}[\text { dare-ga utau }] \rrbracket &
\end{array}
$$

Applying ! to a wh-clause yields a tautology. Thus, the at-issue meaning of (48) is tautologous. The non-at-issue meaning conveys that the speaker is entertaining the issue who will sing. Just as in the non- $w h$ case this non-at-issue meaning is compatible with the speaker believing a specific answer to the question. Yet, the sentence has an ignorance implication due to the presence of more informative alternatives where the wh-phrase is replaced with a particular name, e.g., Taro-ga utau darou $\downarrow$.

The version of (48) with $k a$ is predicted to have the same semantic values as (49) since the semantic contribution of $k a$, i.e., $\langle ?\rangle$, is vacuous when it is applied to an inquisitive clause, as shown below:

$$
\begin{aligned}
& \text { a. } \llbracket \text { dare-ga utau darou ka } \downarrow \rrbracket=\llbracket !\langle ?\rangle !\langle ?\rangle[\text { dare-ga utau }] \rrbracket=(49 \mathrm{a}) \\
& \text { b. } \quad \llbracket \text { dare-ga utau darou } \mathrm{ka} \downarrow \downarrow \rrbracket^{\bullet}=\llbracket E_{\odot}\langle ?\rangle[\text { dare-ga utau }] \rrbracket=(49 \mathrm{~b})
\end{aligned}
$$

Wh-clause + darou $(-k a) \uparrow \quad$ If we replace the fall in (48) with a rise, the sentence becomes unacceptable, as exemplified in the following:

$$
\text { *Dare-ga utau darou(-ka) } \uparrow
$$

Just as in the case of non- $w h$-cases discussed in $\$ 4.2 .1$, this fact receives a blocking-

7 We thank an anonymous SALT reviewer for pointing out to us that $k a$ is optional in (48). 
based account since the semantic values of (51) would be equivalent to the ones for (48), as shown below:

$$
\begin{array}{lll}
\text { a. } & \text { dare-ga utau darou } \uparrow \rrbracket & =\llbracket\langle ?\rangle\langle ?\rangle ![\text { dare-ga utau }] \rrbracket \quad \text { (tautologous) } \\
\text { b. } & \llbracket \text { dare-ga utau darou } \uparrow \rrbracket & =\llbracket E_{\odot}[\text { dare-ga utau }] \rrbracket
\end{array}
$$

That is, given the equivalence between (49) and (52), the assumption that a sentencefinal rise is more marked than a sentence-final fall predicts that (51) is blocked by (48) (to be more precise, (51)-with- $k a$ is blocked by (48)-with- $k a$, and (51)-without$k a$ by (48)-without- $k a$.)

One remaining question is why the version of (48) with $k a$ is not blocked by the version without, despite their semantic equivalence and the apparent lower syntactic complexity of the version without $k a$. A possible answer to this question would be that dare must be licensed either by a local $k a$ or $m o$ (in which case it is interpreted as an existential or a universal quantifier, respectively), or by a sentence-final interrogative marker which is optionally spelled out as $\mathrm{ka}$. On this account, (48) without $k a$ still contains a sentence-final interrogative marker and is therefore not syntactically less complex than (48) with $k a$. This would predict the absence of blocking. ${ }^{8}$

\section{Conclusions}

We have pointed out that the interrogative-embedding use of darou cannot be reduced to its declarative-embedding use. This places darou in the same class as other modal operators that have recently been argued to require a non-reductive analysis (George 2011; Elliott et al. 2017; Roberts 2018). Such cases are problematic under the standard assumption that modal operators always apply to propositions. We have developed a detailed account of darou, capturing its non-reductive nature as well as its puzzling interaction with intonation. The account crucially rests on the assumption that declarative and interrogative clauses denote the same kind of semantic object, namely sets of propositions, and that modal operators apply uniformly to both kinds of clauses, taking such sets of propositions as their input.

8 As mentioned in footnote 2, shortly before finalising the current paper we became aware of Hara's recent work on darou (Hara 2017, 2018). While a detailed comparison of the two approaches must be left for future work, we highlight some salient differences here. First, we predict that $p$-darou $\downarrow$ has a non-at-issue implication that the speaker believes $p$, and that $p$-darou-ka $\downarrow$ has a non-at-issue implication that the speaker wonders whether $p$. Hara predicts that these are at-issue implications. We think that this is problematic in view of our observations in (13) and (37). Second, we predict that $p$-darou $\uparrow$ is semantically inquisitive. For Hara, it is semantically non-inquisitive. Rather, it is semantically equivalent to 'I believe $p$ ' with a final rise. We find this approach less attractive because the latter construction is infelicitous in Japanese. Without further stipulations, one would thus incorrectly predict $p$-darou $\uparrow$ to be infelicitous as well. Third, the present account covers not only cases in which darou takes a polar question as its prejacent, but also cases with wh-questions. 
Do modals take propositions or sets of propositions? Evidence from Japanese darou

\section{References}

Blutner, Reinhard. 2000. Some aspects of optimality in natural language interpretation. Journal of Semantics 17(3). 189-216.

Ciardelli, Ivano. 2016. Questions in logic. Amsterdam: Institute of Logic, Language and Computation, University of Amsterdam PhD dissertation.

Ciardelli, Ivano \& Floris Roelofsen. 2015. Inquisitive dynamic epistemic logic. Synthese 192(6). 1643-1687.

Ciardelli, Ivano \& Floris Roelofsen. 2018. An inquisitive perspective on modals and quantifiers. Annual Review of Linguistics 4. 129-149. doi:10.1146/annurevlinguistics-011817-045626.

Dayal, Veneeta. 1996. Locality in Wh-quantification: Questions and Relative Clauses in Hindi. Kluwer Academic Publishers. doi:10.1007/978-94-011-48085.

Elliott, Patrick D., Nathan Klinedinst, Yasutada Sudo \& Wataru Uegaki. 2017. Predicates of relevance and theories of question embedding. Journal of Semantics 34(3). 547-554. doi:10.1093/jos/ffx008.

George, B.R. 2011. Question embedding and the semantics of answers. Los Angeles, CA: University of California, Los Angeles PhD dissertation.

George, B.R. 2013. Knowing-wh, mention-some readings, and non-reducibility. Thought: A Journal of Philosophy 2(2). 166-177.

Gussenhoven, Carlos. 2002. Intonation and interpretation: phonetics and phonology. In The 1st international conference on speech prosody, 47-57.

Hamblin, Charles L. 1973. Questions in Montague English. Foundations of Language 10(1). 41-53.

Hara, Yurie. 2006. Grammar of knowledge representation: Japanese discourse items at interfaces. Newark, DE: University of Delaware PhD dissertation.

Hara, Yurie. 2015. Darou ka: an interplay of bias, sentence types, and prosody. Ms., City University of Hong Kong, available online at Semantics Archive.

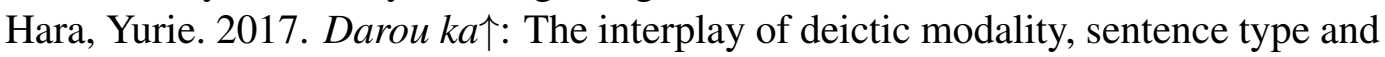
prosody. Ms., Waseda University, available online at Semantics Archive.

Hara, Yurie. 2018. Daroo as an entertain modal: an inquisitive approach. The 25th Japanese/Korean linguistics (JK).

Hara, Yurie \& Chris Davis. 2013. Darou as a deictic context shifter. In Kazuko Yatsushiro \& Uli Sauerland (eds.), Formal Approaches to Japanese Linguistics (FAJL) 6, 41-56. MITWPL.

Heim, Irene. 1994. Interrogative semantics and Karttunen's semantics for know. In R. Buchalla \& A. Mittwoch (eds.), The Ninth Annual Conference and the Workshop on Discourse of the Israel Association for Theoretical Linguistics (IATL), Jerusalem: Academon. 
Horn, Laurence R. 1984. Towards a new taxonomy of pragmatic inference: Qbased and R-based implicatures. In D. Schiffrin (ed.), Meaning, Form, and Use in Context, 11-42. Georgetown University Press.

Karttunen, Lauri. 1977. Syntax and semantics of questions. Linguistics and Philosophy 1. 3-44. doi:10.1007/bf00351935.

Karttunen, Lauri \& Stanley Peters. 1976. What indirect questions conventionally implicate. In Chicago Linguistic Society (CLS) 12, 351-368. Chicago Linguistic Society.

Karttunen, Lauri \& Stanley Peters. 1979. Conventional implicature. Syntax and Semantics 11. 1-56.

Katzir, Roni. 2007. Structurally-defined alternatives. Linguistics and Philosophy 30(6). 669-690. doi:10.1007/s10988-008-9029-y.

Kratzer, Angelika. 1981. The notional category of modality. In H.J. Eikmeyer \& H. Rieser (eds.), Words, Worlds, and Contexts: New Approaches in Word Semantics, 38-74. Berlin: Walter de Gruyter.

Lahiri, Utpal. 2000. Lexical selection and quantificational variability in embedded interrogatives. Linguistics and Philosophy 23(4). 325-389.

Levinson, Stephen. 2000. Presumptive Meanings. Cambridge, MA: MIT press.

Morimoto, Junko. 1994. Hanashite no shukan o arawasu fukushi ni tsuite [On adverbs that represent the speaker's subjectivity]. Tokyo: Kuroshio.

Roberts, Tom. 2018. Responsive predicates are question-embedding: Evidence from Estonian. In Sinn und Bedeutung 22, 271-288.

Roelofsen, Floris \& Donka F. Farkas. 2015. Polarity particle responses as a window onto the interpretation of questions and assertions. Language 91(2). 359-414.

Spector, Benjamin \& Paul Egré. 2015. A uniform semantics for embedded interrogatives: An answer, not necessarily the answer. Synthese 192(6). 1729-1784.

Steinert-Threlkeld, Shane. to appear. An explanation of veridical uniformity universal. Journal of Semantics .

Sugimura, Yasushi. 2004. Gaizensei o arawasu fukushi to bunmatsu no modality keishiki [Adverbs of probability and sentence-final modality expressions]. Gengo Bunka Ronshuu 25(2). 90-111.

Takubo, Yukinori. 2001. Gendai nihongo ni okeru nishu no modal jodoshi ni tsuite [On two kinds of modal auxiliaries in Modern Japanese]. Kannichigo Bungaku Ronsou 1003-1025.

Theiler, Nadine, Floris Roelofsen \& Maria Aloni. 2018. A uniform semantics for declarative and interrogative complements. Journal of Semantics 35(3). 409466. doi:10.1093/jos/ffy003.

Uegaki, Wataru. 2015. Interpreting questions under attitudes. Cambridge, MA: Massachusetts Institute of Technology PhD dissertation.

Uegaki, Wataru. to appear. The semantics of question-embedding predicates. Lan- 
Do modals take propositions or sets of propositions? Evidence from Japanese darou

guage and Linguistics Compass .

Westera, Matthijs. 2017. Exhaustivity and intonation: a unified theory. Amsterdam: Institute for Logic, Language and Computation, University of Amsterdam PhD dissertation.

Wataru Uegaki

Van Wijkplaats 4

2311 BX Leiden

Netherlands

w.uegaki@hum.leidenuniv.nl
Floris Roelofsen

P.O.box 94242

1090 GE Amsterdam

Netherlands

floris.roelofsen@gmail.com 